\title{
A CONSENSUS REPORT FROM THE FIRST ASIAN REGIONAL MEETING ON THE TERMINOLOGY AND CRITERIA FOR VERRUCO-PAPILLARY LESIONS OF THE ORAL CAVITY HELD IN KUALA LUMPUR, MALAYSIA, DECEMBER 15-18, 2013
}

R.B. Zain ${ }^{1}$, Thomas George Kallarakkal', Anand Ramanathan $^{1}$, Jin Kim², W.M. Tilakaratne, Takashi Takata $^{4}$, Saman Warnakulasuriya ${ }^{5}$ Vinay Kumar Hazarey, Alison Rich ${ }^{7}$, Haizal Mohd Hussaini ${ }^{8}$, Ajura Jalil ${ }^{9}$ and an International Participating Group $(I P G)^{10}$. A Consensus Report from the First Asian Regional Meeting on the Terminology and Criteria for Verruco-papillary Lesions of the Oral Cavity held in Kuala Lumpur, Malaysia, December 15-18, 2013. Annal Dent Univ Malaya 2013; 20(2): $1-3$

1. Oral Cancer Research and Coordinating Centre \& Department of Oro-Maxillofacial Surgical and Medical Sciences, Faculty of Dentistry, University of Malaya, Kuala Lumpur, Malaysia

2. Department of Oral Pathology, Oral Cancer Research Institute , Yonsei University College of Dentistry, Seoul, Korea

3. Centre for Research in Oral Cancer, Faculty of Dental Sciences, University of Peradeniya, Sri Lanka

4. Department of Oral and Maxillofacial Pathobiology, Institute of Biomedical and Health Sciences, Hiroshima University, Hiroshima, Japan

5. Department of Oral Medicine \& Pathology, Guy's, King's and St Thomas' Dental Institute, WHO Collaborating Centre for Oral Cancer and Precancer, London, United Kingdom

6. Department of Oral Pathology and Microbiology, Government Dental College and Hospital, Nagpur, India

7. Department of Oral Diagnostic and Surgical Sciences, Dental School Health sciences, University of Otago, New Zealand

8. Department of Oral Pathology and Oral Medicine, Faculty of Dentistry, University Kebangsaan Malaysia, Kuala Lumpur, Malaysia

9. Stomatology Unit, Institute for Medical Research, Kuala Lumpur, Malaysia

10. *Contributing Participants at the Meeting (names \& affiliations at the end of the text)

Verruco-papillary lesions (VPLs) of the oral cavity described in the literature involve a spectrum of conditions including squamous papilloma, verruca vulgaris, focal epithelial hyperplasia, condyloma, proliferative verrucous leukoplakia and verrucous carcinoma. The majority of the VPLs are slow growing, benign in nature and have a viral aetiology (1). Mucosal HPV types (HPV 6, 11, 13, 30,

\section{Brief Report}

$32,45,52,55,59,69,72$ and 73) have been implicated as possible etiological causes for these benign lesions (2) while virus associated benign mucosal outgrowths are not too difficult to diagnose either clinically or by microscopy. Apart from virus-associated lesions, VPLs harboring malignant potential such as verrucous carcinoma, proliferative verrucous leukoplakia and oral verrucous hyperplasia $(\mathrm{OVH})$ need to be further clarified for better understanding of their predictable biologic behavior and appropriate treatment. In particular, the condition referred to as oral verrucous hyperplasia $(\mathrm{OVH})$ poses a major diagnostic challenge. OVH represents a histopathological entity whose clinical features are not well recognised and is usually clinically indistinguishable from a verrucous carcinoma (3).

In 1980, Shear and Pindborg classified OVHs into two clinical variants, a sharp variety comprising of long, narrow, heavily keratinized verrucous processes which appears white as a result of heavy keratinization and a second variant referred to as the blunt variety consisting of verrucous processes that are broader, flatter and not heavily keratinized (3). A new pathological entity distinct from what Shear and Pindborg earlier described has been found in recent years among betel-quid chewers mainly from Taiwan. In 2005, Chung et al., in a field survey of 1075 adults noted 9 verrucous lesions which they described as exophytic outgrowths, which the authors hinted had hitherto not been reported in the scientific literature (4). Their Figure: 1 illustrated this newly described "verrucous lesion". Subsequently in 2009 Wang et al described a case series of 60 cases from Taipei and classified these lesions as plaque-type and mass-type lesions primarily based on their histopathological features. It was also documented that the mass-type verrucous hyperplasia may manifest as single or multiple verrucous whitish pink lesions clinically while the plaque-type lesions may appear as whitish verrucous plaques. They also concluded that the terminology $\mathrm{OVH}$ should be reserved to denote only the mass-type lesions both clinically and histologically and suggested that the plaque-type lesions should be clinically classified as oral verruciform leukoplakia and histologically as verruciform hyperplasia (5).

In an effort to bring uniformity in reporting these lesions both clinically and histopathologically a consensus meeting was held in Kuala lumpur, Malaysia during December 15-18, 2013. A working committee that included specialists working on oral malignant and 
potentially malignant disorders attempted to formulate the clinical and histopathological criteria of $\mathrm{OVH}$ based on the discussion among the participants in the meeting. The meeting was attended by 46 participants from 7 countries and included specialists and trainees in the disciplines of Oral Medicine and Oral and Maxillofacial Pathology. Consensus guidelines arising from this meeting is as follows.

\section{CLINICAL CRITERIA FOR VERRUCOUS HYPERPLASIA OF THE ORAL CAVITY:}

The working committee proposed the term "Exophytic Verrucous Hyperplasia" to denote the clinical entity that represents the microscopic diagnosis of OVH. The following criteria for the clinical diagnosis of OVH was proposed:

a. These lesions clinically present in two forms: 1) as an exophytic, fleshy verruco-papillary outgrowth with a white and/or pink surface color and 2) as a white, plaque-like exophytic verrucous lesion. The latter may mimic verrucous leukoplakia. In both instances the clinical term 'exophytic verrucous hyperplasia' should be used.

b. Exophytic verrucous hyperplasia may occur in any anatomical site in the oral cavity and in general would be more than $1 \mathrm{~cm}$ in size.

c. Unlike proliferative verrucous leukoplakia (PVL) exophytic verrucous hyperplasia is a discrete and solitary lesion.

d. Exophytic verrucous hyperplasia may co-exist in a patient presenting with oral submucous fibrosis.

e. The clinical presentation of exophytic verrucous hyperplasia could masquerade as a squamous cell carcinoma or verrucous carcinoma. Absence of induration is a cardinal feature of exophytic verrucous hyperplasia.

\section{HISTOLOGICAL CRITERIA FOR VERRUCOUS HYPERPLASIA OF THE ORAL CAVITY}

The working committee proposed the following criteria for the histological diagnosis of oral verrucous hyperplasia:

a. Keratinized exophytic verruco-papillary processes seen. Keratin plugging may be present.

b. Epithelium is hyperplastic with both basal cell hyperplasia and acanthosis.

c. Absence of downward growth of the hyperplastic epithelium into the lamina propria when compared with the level of the basement membrane of the adjacent normal epithelium.

d. Epithelial dysplasia may or may not be present.

e. Subepithelial lymphocytic infiltration as a host response may or may not be present.

f. Verrucous hyperplasia should be clearly differentiated from verrucous carcinoma which exhibits frank downward growth of the epithelial processes below the level of the basement membrane of the adjacent normal epithelium.

g. Verrucous hyperplasia should be differentiated from squamous cell papilloma by its size and by the presence of a prominent fibrovascular core in the latter.

h. In a small biopsy without adjacent normal mucosal epithelium particular attention should be paid to exclude other pathologies such as squamous cell papilloma and verrucous carcinoma.

It was proposed by the working committe that of above, criteria (a), (b) and (c) must be present to make a histopathological diagnosis of OVH. Further it was recommended that the pathology report should include a statement describing the degree of dysplasia if present and a cautionary note to say that $\mathrm{OVH}$ may recur following excision and may progress to verrucous carcinoma and squamous cell carcinoma. Therefore careful surveillance is mandatory.

\section{CONCLUSIONS}

The terminology and the criteria proposed by the working committee at this consensus meeting will strengthen the understanding of what is already known and will serve to minimize the use of conflicting terminologies that designate $\mathrm{OVH}$. The malignant potential of $\mathrm{OVH}$ has been shown in a number of publications $(5,6,7,8)$. Future studies in South Asia should examine the natural history of this novel disorder and we propose that it should be considered as one of the oral potentially malignant disorders at the next WHO revision of classification and nomenclature of Oral Potentially Malignant disorders (9). We may not succeed in completely eliminating the use of these varied terminologies but a consensus for a universal proposed guidelines and terminologies can be further validated in future case series.

\section{REFERENCES}

1. Thomas GJ, Barrett AW (2009) Papillary and verrucous lesions of the oral mucosa. Diagnostic Histopathology, 15(6), 279-285. 
2. Terai M, Takagi M (2001) Human papillomavirus in the oral cavity. Journal of Oral Medicine \& Pathology, 6(1), 1-12.

3. Shear M, Pindborg JJ (1980) Verrucous hyperplasia of the oral mucosa. Cancer, 46(8), 1855-1862.

4. Chung, C. H., Yang, Y. H., Wang, T. Y., Shieh, T. Y., \& Warnakulasuriya, S. (2005). Oral precancerous disorders associated with areca quid chewing, smoking, and alcohol drinking in southern Taiwan. Journal of oral pathology \& medicine, 34(8), 460466.

5. Wang YP, Chen HM, Kuo RC, Yu CH, Sun A, Liu BY, Kuo YS, Chiang CP (2009) Oral verrucous hyperplasia: histologic classification, prognosis, and clinical implications. Journal of Oral Pathology \& Medicine, 38(8), 651-656.

6. Chen, H. M., Chen, C. T., Yang, H., Kuo, M. Y. P., Kuo, Y. S., Lan, W. H., ... \& Chiang, C. P. (2004). Successful treatment of oral verrucous hyperplasia with topical 5-aminolevulinic acid-mediated photodynamic therapy. Oral oncology, 40(6), 630637.

7. Klieb, H. B. E., \& Raphael, S. J. (2007). Comparative study of the expression of p53, Ki67, E-cadherin and MMP-1 in verrucous hyperplasia and verrucous carcinoma of the oral cavity. Head and neck pathology, 1(2), 118-122.

8. Yu, C. H., Chen, H. M., Hung, H. Y., Cheng, S. J., Tsai, T., \& Chiang, C. P. (2008). Photodynamic therapy outcome for oral verrucous hyperplasia depends on the clinical appearance, size, color, epithelial dysplasia, and surface keratin thickness of the lesion. Oral oncology, 44(6), 595-600.

9. Warnakulasuriya, S., Johnson, N., \& Van der Waal, I. (2007). Nomenclature and classification of potentially malignant disorders of the oral mucosa. Journal of oral pathology \& medicine, 36(10), 575580 .

\section{*International Participating Group(IPG):}

Abd. Rahim B. Ahmad, Hospital Tengku Ampuan Afzan, Kuantan, Ministry of Health (MOH) Malaysia; Ajay Telang, Department of Oral Pathology, Penang International Dental College, Penang, Malaysia; ChingYi- Chen, School of Dentistry, Kaoshiung Medical University, Taiwan; Hartinie Muhamad, Hospital Raja Perampuan Zainab II MOH, Kota Bharu, Kelantan, Malaysia; Hans Prakash a/l Sathasivam, Hospital Sultan Ismail, MOH, Johor Bahru, Malaysia; Lau Shin Hin Stomatology Unit, Institute for Medical Research, $\mathrm{MOH}$, Kuala Lumpur, Malaysia; Ikuko Ogawa, Centre of Oral Clinical Examination, Hiroshima University Hospital, Hiroshima University, Hiroshima, Japan Liana Ma Abdullah, Ministry of Defence, Kuala Lumpur, Malaysia; Masitah Hayati Harun, Department of Oral Pathology and Oral Medicine, School of Dental Sciences, Universiti Sains Malaysia, Kelantan, Malaysia; Nornazaliza Basri, Hospital Raja Permaisuri Bainun, MOH, Perak, Malaysia; Nurhayu Binti Ab. Rahman, Department of Oral Pathology and Oral Medicine, School of Dental Sciences, Universiti Sains Malaysia, Kelantan, Malaysia; Nurshaline Pauline Kipli, Hospital Umum Sarawak, Sarawak, Malaysia; Siar Chong Huat, Department of Oro-maxillofacial Surgical and Medical Sciences, Faculty of Dentistry, University of Malaya, Kuala Lumpur, Malaysia; Suraya Hani Mohd Sinon, Department of Oral Pathology and Oral Medicine, Faculty of Dentistry, Universiti Kebangsaan Malaysia, Kuala Lumpur, Malaysia; Sumairi Bt. Ismail, Hospital Sultan Abdul Halim, MOH, Kedah, Malaysia; Sunil Subash Mutalik, School of Dentistry, International Medical University, Malaysia; Vidya G Doddawad, Department of Oral Pathology and Microbiology, JSS Dental College and Hospital, Mysore, India; Vimi Mutalik, School of Dentistry, International Medical University, Malaysia; and Yaghma Masood, Centre of Oral \& Maxillofacial Diagnostics \& Medicine Studies, Faculty of Dentistry, Universiti Teknologi Mara, Malaysia. 